\title{
The Third Spectrum of Gold (Au III)
}

\author{
Laura Iglesias ${ }^{1}$
}

(July 21, 1960)

\begin{abstract}
The spark spectrum of gold has been photographed in a helium atmosphere from $500 \mathrm{~A}$ to $6600 \mathrm{~A}$. About 500 lines have been assigned to the third spectrum, Au III, and separated from those belonging to different stages of ionization, by observation of the polarity of the lines. Sixty two levels have been found: 17 even levels, arising from the $5 d^{9}$ and $5 d^{8} 6 \mathrm{~s}$ configurations; and 45 odd levels, belonging to the $5 d^{8} 6 p$ and $5 d^{7} 6 s 6 p$ configurations. All of the expected levels from the configurations $5 d^{9}, 5 d^{8} 6 s$ and $5 d^{8} 6 p$ have been identified except for the very high terms based on the $5 d^{8}\left({ }^{1} \mathrm{~S}\right)$ core of Au Iv. With these levels it was possible to classify 256 lines.
\end{abstract}

\section{Introduction}

The first and second spectra of gold were studied some years ago, but apparently no attempt has been made to study the structure of the third spectrum, (Au III).

As in most spectra of the third long period, the Au III spectrum reveals a coupling intermediate between $L S$-coupling and $j j$-coupling since the spinspin interaction and the spin-orbit interaction are both large. It approaches $j j$-coupling rather than $L S$, especially in the case of levels belonging to the $5 d^{8} 6 p$ configuration.

It is, therefore, very difficult to describe the observed levels rigorously in any one scheme. Accordingly, the even levels and the odd quartet levels based on $5 d^{8}\left({ }^{3} \mathrm{~F}\right)$, which are perhaps closer to $L S$ coupling, have been given names in that system, and the other odd levels have been given numbers simply for identification.

\section{Analysis and Results}

The analysis is based mainly on plates taken on the $2-m$ vacuum spectrograph at Princeton, in the wavelength region 500 to $2200 \mathrm{~A}$.

The light source was a condensed spark in helium at a pressure of approximately $1 \mathrm{~atm}$.

In order to excite the third spectrum and to be able to differentiate it from the other stages of ionization, the same technique was used that A. G. Shenstone has described in his paper on $\mathrm{Ni}$ III. $^{2}$ Initially, the exposures were limited by the clogging of the slit by gold particles, sputtered from the spark source. Apparently gold has a pronounced tendency to sputter and the complete clogging of the slit took only one or two min, instead of the half hour or more which is commonly found with other metals. To avoid this limitation, it was necessary to fill the body of the spectrograph with helium to atmospheric pressure, so that there would be no flow through the slit. For the spectrograms taken above $1200 \mathrm{~A}$, this was

1 Princeton University, Princeton, N.J.

2 A. G. Shenstone, J. Opt. Soc. Am. 44, 749 (1954). not necessary since the lithium fluoride window separated the spectrograph from the source.

The spectrograms were photographed on Ilford Q-2 plates and the exposures varied from 1 to 15 min. The impurity lines of nitrogen, oxygen, and carbon present on the plates were used as standards. The estimated probable error of the experimental wavelengths entered in table 1 , column 1 , is $\pm 0.01 \mathrm{~A}$.

To complete the observations, other plates were taken on the 21-ft grating in a Wadsworth mounting by using Eastman $103 \mathrm{aO}$ plates, $103 \mathrm{aD}$, and $103 \mathrm{aF}$ according to the region. About 500 lines in all were assigned to the third spectrum.

The lowest levels should be the ${ }^{2} \mathrm{D}_{21 / 2}$ and $\mathrm{D}_{11 / 2}$ of the $5 d^{9}$ cofinguration, and by comparison with the $\mathrm{Au}$ I and $\mathrm{Au}$ II spectra, ${ }^{3}$ the interval between them should be about $-13000 \mathrm{~cm}^{-1}$. The equivalent interval in $\mathrm{Au} \mathrm{I}$ and $\mathrm{II}$ is as follows:

$$
\begin{aligned}
& \mathrm{Au} \text { I } \quad 5 d^{9} 6 s^{2} \quad{ }^{2} \mathrm{D}_{21 / 2}-{ }^{2} \mathrm{D}_{11 / 2}-12274 \\
& \mathrm{Au} \text { II } \quad 5 d^{9}\left({ }^{2} \mathrm{D}\right) 6 s \quad{ }^{3} \mathrm{D}_{3}-{ }^{3} \mathrm{D}_{1} \quad-12725
\end{aligned}
$$

In fact, we found it to be -12694.0 in Au III.

In order to have some idea about the position of the levels of the $5 d^{8} 6 s$ and $5 d^{8} 6 p$ configurations we plotted Ir I and Pt II and the analogous spectra of the second long period Rh I, Pd II and Ag III, using the values given in "Atomic Energy Levels". ${ }^{3}$ To find the levels the usual method of searching for equal wave-number differences was used.

A remarkable similarity was found in the case of the terms arising from the $5 d^{8} 6 s$ configuration. This relationship is shown in figure 1 where the relative values of the levels of Ir I, Pt II and Au III are plotted against $J$-values.

Based on this similarity and the reason explained above, a tentative $L S$-term assignment has been made and is given in table 2, where all the known even levels are arranged in increasing numerical order.

In the case of the odd configuration $5 d^{8} 6 p$, the levels are so mixed in character that it is meaningless to group them into terms. A very tentative designation based on combinations and intensities appears

\footnotetext{
3 C. E. Moore, A tomic Energy Levels, NBS Circ. 467 (1958).
} 


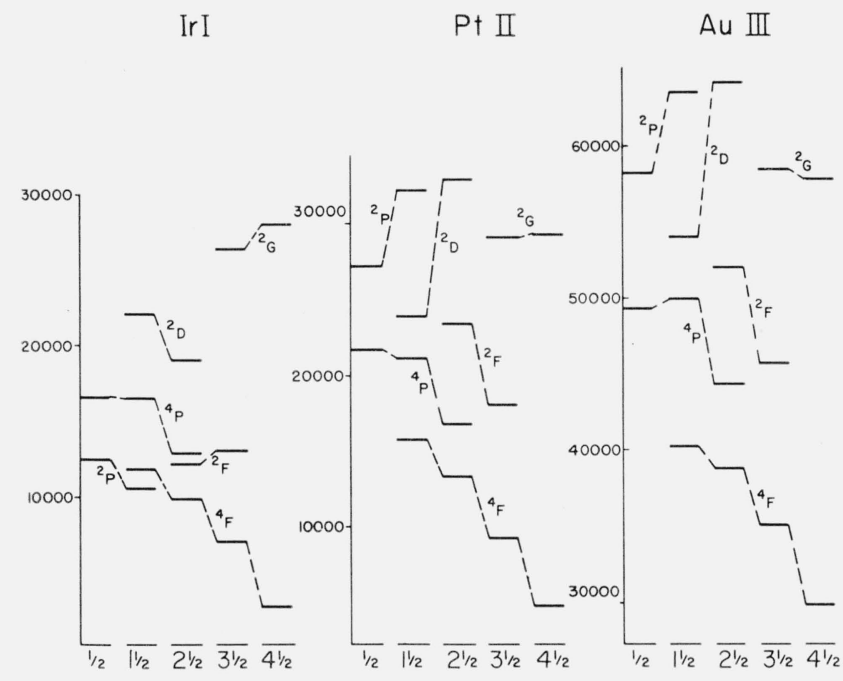

Figure 1. Configuration $5 d^{8} 6 s$ in $\mathrm{Ir}$ I, PtiI, and AuirI. in table 3 , but these names, except for the quartet levels based on $5 d^{8}\left({ }^{3} \mathrm{~F}\right)$, are not used in the line list of table 1 . Instead, the levels are assigned numbers for identification.

In table 3,43 levels of the $5 d^{8} 6 p$ configuration are listed, plus two more belonging to the $5 d^{7} 6 s 6 p$ configuration.

The configurations $5 d^{9}, 5 d^{8} 6 s$ and $5 d^{8} 6 p$ are complete except for the usual failure to find the levels based on $5 d^{8}\left({ }^{1} \mathrm{~S}\right)$ of $\mathrm{Au} \mathrm{IV}$.

With the 62 levels found, it was possible to classify 256 of the 500 observed lines attributable to $\mathrm{Au}$ III as they appear in table 1 .

The author expresses gratitude to Professor A. G. Shenstone for his suggestion of this research and for his assistance during the course of the work. My thanks are also due to Princeton University which made my stay in Princeton possible through an appointment as a Research Assistant on the Eugene Higgins Fund.

TABLE 1. Identified lines of Au III

\begin{tabular}{|c|c|c|c|c|c|c|c|}
\hline$\lambda$ (vac.) & $\begin{array}{c}\text { Intensity } \\
\text { and } \\
\text { character }\end{array}$ & Wave No. & Combination & $\lambda$ (vac.) & $\begin{array}{l}\text { Intensity } \\
\text { and } \\
\text { character }\end{array}$ & Wave No. & Combination \\
\hline $\begin{array}{c}A \\
751.554 \\
763.497 \\
779.728 \\
788.783 \\
811.394\end{array}$ & $\begin{array}{r}2 \\
20 \\
30 \\
30 \\
25\end{array}$ & $\begin{array}{c}\mathrm{cm}^{-1} \\
133057.6 \\
130976.3 \\
128249.8 \\
126777.6 \\
123244.7\end{array}$ & $\begin{array}{l}a{ }^{2} \mathrm{D}_{21 / 2}-42_{21 / 2}^{\circ} \\
a^{2} \mathrm{D}_{21 / 2}-40_{31 / 2}^{\circ} \\
a^{2} \mathrm{D}_{21 / 2}-38_{11 / 2}^{112} \\
a{ }^{4} \mathrm{~F}_{41 / 2}-44_{31 / 2}^{\circ} \\
a^{4} \mathrm{~F}_{31 / 2}-45_{21 / 2}^{\circ}\end{array}$ & \begin{tabular}{r}
\multicolumn{1}{c}{$A$} \\
977.318 \\
989.408 \\
998.156 \\
1034.206 \\
1040.650
\end{tabular} & $\begin{array}{r}40 \\
25 \\
20 \\
60 \\
100\end{array}$ & $\begin{array}{r}c m^{-1} \\
102320.8 \\
101070.5 \\
100184.7 \\
96692.5 \\
96093.8\end{array}$ & $\begin{array}{l}a^{2} \mathrm{D}_{21 / 2}-z^{4} \mathrm{G}_{31 / 2}^{\circ} \\
a^{2} \mathrm{D}_{11 / 2}-19_{01 / 2}^{\circ} \\
a^{2} \mathrm{D}_{11 / 2}-z^{4} \mathrm{~F}_{21 / 2}^{\circ} \\
a^{2} \mathrm{D}_{11 / 2}-z^{4} \mathrm{~F}_{11 / 2}^{\circ} \\
a^{2} \mathrm{D}_{21 / 2}-z^{4} \mathrm{G}_{21 / 2}^{\circ}\end{array}$ \\
\hline $\begin{array}{l}811.831 \\
816.129 \\
817.96 \\
820.053 \\
820.846\end{array}$ & $\begin{array}{r}40 \\
3 \\
50 \\
40 \\
5\end{array}$ & $\begin{array}{l}123178.3 \\
122529.6 \\
122256 \\
121943.3 \\
121825.5\end{array}$ & $\begin{array}{l}a^{2} \mathrm{D}_{21 / 2}-32_{11 / 2}^{\circ} \\
a^{2} \mathrm{D}_{21 / 2}-31_{21 / 2}^{112} \\
a^{2} \mathrm{D}_{11 / 2}-43_{21 / 2}^{1} \\
a^{2} \mathrm{D}_{21 / 2}-29_{11 / 2}^{\circ} \\
a^{2} \mathrm{D}_{21 / 2}-28_{31 / 2}^{\circ}\end{array}$ & $\begin{array}{l}\text { 1044. } 497 \\
1046.825 \\
1054.192 \\
1086.110 \\
1123.172\end{array}$ & $\begin{array}{r}80 \\
80 \\
40 \\
10 \\
5\end{array}$ & $\begin{array}{l}95739.9 \\
95526.9 \\
94859.4 \\
92072.5 \\
89033.5\end{array}$ & $\begin{array}{l}a^{2} \mathrm{D}_{21 / 2}-z^{4} \mathrm{D}_{11 / 2}^{\circ} \\
a^{2} \mathrm{D}_{11 / 2}-14_{21 / 2}^{\circ} \\
a^{2} \mathrm{D}_{11 / 2}-13_{21 / 2}^{\circ} \\
a^{4} \mathrm{~F}_{41 / 2}-28_{31 / 2}^{\circ} \\
a^{2} \mathrm{D}_{11 / 2}^{\circ}-z^{4} \mathrm{D}_{21 / 2}^{\circ}\end{array}$ \\
\hline $\begin{array}{l}823.338 \\
833.149 \\
836.804 \\
843.454 \\
845.138\end{array}$ & $\begin{array}{r}10 \\
80 \\
15 \\
100 \\
100\end{array}$ & $\begin{array}{l}121456.8 \\
120026.5 \\
119502.3 \\
118560.1 \\
118323.9\end{array}$ & $\begin{array}{l}a{ }^{4} \mathrm{~F}_{31 / 2}-44_{31 / 2}^{\circ} \\
a^{2} \mathrm{D}_{21 / 2}-27_{21 / 2}^{\circ} \\
a^{4} \mathrm{~F}_{21 / 2}-45_{21 / 2}^{\circ} \\
a^{2} \mathrm{D}_{21 / 2}-26_{11 / 2}^{\circ} \\
a^{2} \mathrm{D}_{21 / 2}-25_{31 / 2}^{\circ}\end{array}$ & $\begin{array}{l}\text { 1199. } 022 \\
1204.155 \\
1229.364 \\
1231.060 \\
1231.266\end{array}$ & $\begin{array}{l}25 \\
30 \\
30 \\
20 \\
30\end{array}$ & $\begin{array}{l}83401.3 \\
83045.8 \\
81342.9 \\
81230.8 \\
81217.2\end{array}$ & $\begin{array}{l}a{ }^{2} \mathrm{D}_{11 / 2}-z^{4} \mathrm{G}_{21 / 2}^{\circ} \\
a^{2} \mathrm{D}_{11 / 2}-z^{4} \mathrm{D}_{11 / 2}^{\circ} \\
a{ }^{4} \mathrm{P}_{21 / 2}-34_{11 / 2}^{\circ} \\
a^{4} \mathrm{~F}_{41 / 2}^{\circ}-16_{31 / 2}^{\circ} \\
a^{4} \mathrm{~F}_{31 / 2}-23_{31 / 2}^{\circ}\end{array}$ \\
\hline $\begin{array}{l}847.619 \\
849.546 \\
855.495 \\
859.891 \\
863.425\end{array}$ & $\begin{array}{r}5 \\
5 \\
80 \\
80 \\
80\end{array}$ & $\begin{array}{l}117977.5 \\
117709.9 \\
116891.4 \\
116293.8 \\
115817.8\end{array}$ & $\begin{array}{l}a{ }^{4} \mathrm{~F}_{11 / 2}-45_{21 / 2}^{\circ} \\
a{ }^{4} \mathrm{~F}_{21 / 2}-44_{31 / 2}^{\circ} \\
a^{2} \mathrm{D}_{21 / 2}-24_{11 / 2}^{\circ} \\
a^{2} \mathrm{D}_{21 / 2}-23_{31 / 2}^{\circ} \\
a^{2} \mathrm{D}_{11 / 2}-39_{21 / 2}^{\circ}\end{array}$ & $\begin{array}{l}1239.961 \\
1254.996 \\
1277.442 \\
1278.514 \\
1280.903\end{array}$ & $\begin{array}{c}100 \mathrm{w} \\
30 \\
5 \\
100 \\
50\end{array}$ & $\begin{array}{l}80647.7 \\
79681.5 \\
78281.4 \\
78215.8 \\
78069.9\end{array}$ & $\begin{array}{ll}a & { }^{4} \mathrm{~F}_{31 / 2}-22_{41 / 2}^{\circ} \\
a & { }^{4} \mathrm{~F}_{11 / 2}-27_{21 / 2}^{\circ} \\
a & { }^{4} \mathrm{P}_{11 / 2}-38_{11 / 2}^{\circ} \\
a & { }^{4} \mathrm{~F}_{11 / 2}-26_{11 / 2}^{\circ} \\
a & { }^{4} \mathrm{~F}_{21 / 2}-24_{11 / 2}^{\circ}\end{array}$ \\
\hline $\begin{array}{l}883.782 \\
885.906 \\
901.025 \\
905.105 \\
910.446\end{array}$ & $\begin{array}{l}50 \\
60 \\
80 \\
30 \\
80\end{array}$ & $\begin{array}{l}113150.1 \\
112878.8 \\
110984.6 \\
110484.4 \\
109836.3\end{array}$ & $\begin{array}{l}a^{2} \mathrm{D}_{11 / 2}-35_{01 / 2}^{\circ} \\
a^{2} \mathrm{D}_{21 / 2}-z^{4} \mathrm{~F}_{21 / 2}^{\circ} \\
a^{2} \mathrm{D}_{21 / 2}-16_{31 / 2}^{\circ} \\
a^{2} \mathrm{D}_{11 / 2}^{\circ}-32_{11 / 2}^{\circ} \\
a^{2} \mathrm{D}_{11 / 2}-31_{21 / 2}^{\circ}\end{array}$ & $\begin{array}{l}1285.302 \\
1290.029 \\
1290.358 \\
1290.795 \\
1291.979\end{array}$ & $\begin{array}{l}50 \\
30 \\
40 \\
50 \\
60\end{array}$ & $\begin{array}{l}77802.7 \\
77517.6 \\
77497.9 \\
77471.6 \\
77400.6\end{array}$ & $\begin{array}{ll}a & { }^{4} \mathrm{~F}_{31 / 2}-z^{4} \mathrm{~F}_{21 / 2}^{\circ} \\
a & { }^{4} \mathrm{P}_{21 / 2}-29_{11 / 2}^{\circ} \\
a & { }^{4} \mathrm{P}_{11 / 2}-37_{11 / 2}^{\circ} \\
a & { }^{4} \mathrm{~F}_{21 / 2}-23^{\circ}{ }^{\circ} 1 / 2 \\
a & { }^{4} \mathrm{P}_{21 / 2}-28_{31 / 2}^{\circ}\end{array}$ \\
\hline $\begin{array}{l}911.470 \\
914.175 \\
915.338 \\
924.037 \\
931.675\end{array}$ & $\begin{array}{l}20 \\
5 h \\
8 \\
80 \\
10\end{array}$ & $\begin{array}{l}109712.9 \\
109388.2 \\
109249.3 \\
108220.8 \\
107333.6\end{array}$ & $\begin{array}{l}a^{2} \mathrm{D}_{11 / 2}-30_{01 / 2}^{\circ} \\
a^{2} \mathrm{D}_{21 / 2}-z^{4} \mathrm{~F}_{11 / 2}^{\prime} \\
a^{2} \mathrm{D}_{11 / 2}-29_{11 / 2}^{\circ} \\
a^{2} \mathrm{D}_{21 / 2}-14_{21 / 2}^{\circ} \\
a^{2} \mathrm{D}_{11 / 2}-27_{21 / 2}^{\circ}\end{array}$ & $\begin{array}{l}\text { 1306. } 317 \\
\text { 1306. } 409 \\
1307.988 \\
1308.776 \\
1309.440\end{array}$ & $\begin{array}{r}5 \\
5 \\
20 \\
10 \\
40\end{array}$ & $\begin{array}{l}76551.1 \\
76545.7 \\
76453.3 \\
76407.3 \\
76368.5\end{array}$ & $\begin{array}{ll}a & { }^{4} \mathrm{~F}_{21 / 2}-21_{21 / 2}^{\circ} \\
a & 4 \mathrm{~F}_{11 / 2}-24_{11 / 2}^{\circ} \\
a^{2} & \mathrm{~F}_{11 / 2}-39_{21 / 2}^{\circ} \\
a & { }^{4} \mathrm{P}_{01 / 2}-35_{01 / 2}^{\circ} \\
a^{2} \mathrm{G}_{31 / 2}-43_{21 / 2}^{\circ}\end{array}$ \\
\hline $\begin{array}{l}941.050 \\
944.581 \\
945.099 \\
959.720 \\
973.893\end{array}$ & $\begin{array}{r}20 \\
50 \\
200 \\
15 \\
10\end{array}$ & $\begin{array}{l}106264.3 \\
105867.0 \\
105809.0 \\
104197.0 \\
102680.7\end{array}$ & $\begin{array}{l}a^{2} \mathrm{D}_{21 / 2}-12_{11 / 2}^{\circ} \\
a^{2} \mathrm{D}_{11 / 2}-26_{11 / 2}^{\circ} \\
a^{2} \mathrm{D}_{21 / 2}-z^{4} \mathrm{~F}_{31 / 2}^{\circ} \\
a^{2} \mathrm{D}_{11 / 2}-24_{11 / 2}^{\circ} \\
a^{2} \mathrm{D}_{11 / 2}-21_{21 / 2}^{\circ}\end{array}$ & $\begin{array}{l}\text { 1314. } 825 \\
1322.728 \\
1326.105 \\
1336.700 \\
1341.660\end{array}$ & $\begin{array}{r}100 \\
30 \\
12 \\
200 \\
180\end{array}$ & $\begin{array}{l}76055.7 \\
75601.3 \\
75408.8 \\
74811.1 \\
74534.5\end{array}$ & $\begin{array}{l}a{ }^{4} \mathrm{~F}_{41 / 2}-z^{2}{ }^{4} \mathrm{~F}_{31 / 2}^{\circ} \\
a{ }^{4} \mathrm{P}_{21 / 2}-27^{\circ}{ }^{11 / 2} \\
a^{2} \mathrm{~F}_{21 / 2}-37_{11 / 2}^{\circ} \\
a^{4} \mathrm{~F}_{41 / 2}-z^{4} \mathrm{~F}_{41 / 2}^{\circ} \\
a^{2} \mathrm{G}_{41 / 2}^{\circ}-41_{41 / 2}^{0}\end{array}$ \\
\hline
\end{tabular}


TABLE 1. Identified lines of Au III-Continued

\begin{tabular}{|c|c|c|c|c|c|c|c|}
\hline$\lambda$ (vac.) & $\begin{array}{l}\text { Intensity } \\
\text { and } \\
\text { character }\end{array}$ & Wave No. & Combination & $\lambda$ (vac.) & $\begin{array}{c}\text { Intensity } \\
\text { and } \\
\text { character }\end{array}$ & Wave No. & Combination \\
\hline $\begin{array}{c}A \\
1346.129 \\
1348.873 \\
1349.200 \\
1350.302 \\
1353.200\end{array}$ & $\begin{array}{r}12 \\
100 \\
30 \\
150 \\
50\end{array}$ & $\begin{array}{c}\mathrm{cm}^{-1} \\
74287.1 \\
74136.0 \\
74118.0 \\
74057.5 \\
73898.9\end{array}$ & $\begin{array}{l}a^{2} \mathrm{~F}_{31 / 2}-27_{21 / 2}^{\circ} \\
a^{4} \mathrm{P}_{21 / 2}-26_{11 / 2}^{\circ} \\
b^{2} \mathrm{D}_{11 / 2}-38_{11 / 2}^{\circ} \\
a^{4} \mathrm{~F}_{21 / 2}-z^{4} \mathrm{~F}_{21 / 2}^{\circ}{ }^{11 / 2} \\
a^{4} \mathrm{P}_{21 / 2}-25_{31 / 2}^{\circ}\end{array}$ & $\begin{array}{c}A \\
1502.441 \\
1503.716 \\
1517.049 \\
1528.941 \\
1540.258\end{array}$ & $\begin{array}{r}200 \\
200 \\
10 \\
40 \\
80\end{array}$ & $\begin{array}{l}\mathrm{cm}^{-1} \\
66558.3 \\
66501.9 \\
65917.4 \\
65404.7 \\
64924.2\end{array}$ & $\begin{array}{ll}a & { }^{4} \mathrm{P}_{21 / 2}-16_{31 / 2}^{\circ} \\
a & { }^{2} \mathrm{~F}_{21 / 2}-26_{11 / 2}^{\circ} \\
a & { }^{4} \mathrm{~F}_{11 / 2}-12_{11 / 2}^{\circ} \\
a & { }^{4} \mathrm{P}_{11 / 2}-21_{21 / 2}^{\circ} \\
a^{2} & \mathrm{G}_{31 / 2}-33^{\circ}{ }^{1} 1 / 2\end{array} ?$ \\
\hline $\begin{array}{l}\text { 1355. } 598 \\
1356.109 \\
1362.038 \\
1365.372 \\
1365.949\end{array}$ & $\begin{array}{r}150 \\
150 \\
80 \\
500 \\
10\end{array}$ & $\begin{array}{l}\text { 73768. } 2 \\
73740.4 \\
73419.4 \\
73240.1 \\
73209.2\end{array}$ & $\begin{array}{l}a^{2} \mathrm{G}_{31 / 2}-41_{41 / 2}^{\circ} \\
a^{4} \mathrm{P}_{01 / 2}-32_{11 / 2}^{\circ} \\
a^{4} \mathrm{~F}_{11 / 2}-19_{01 / 2}^{\circ} \\
a^{4} \mathrm{~F}_{41 / 2}-z^{4} \mathrm{G}_{51 / 2}^{\circ} \\
a^{4} \mathrm{P}_{11 / 2}-32_{11 / 2}^{\circ}\end{array}$ & $\begin{array}{l}1541.978 \\
1542.212 \\
1548.473 \\
1554.580 \\
1556.793\end{array}$ & $\begin{array}{r}100 \\
80 \\
100 \\
80 \\
20\end{array}$ & $\begin{array}{l}64851.8 \\
64841.9 \\
64579.7 \\
64326.0 \\
64234.6\end{array}$ & 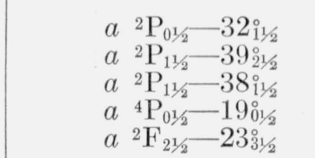 \\
\hline $\begin{array}{l}\text { 1366. } 874 \\
1367.149 \\
1377.708 \\
1378.048 \\
1378.166\end{array}$ & $\begin{array}{r}20 \\
200 \\
180 \\
10 \\
20\end{array}$ & $\begin{array}{l}73159.6 \\
73144.9 \\
72584.3 \\
72566.4 \\
72560.2\end{array}$ & $\begin{array}{ll}a^{2} \mathrm{G}_{41 / 2}-40_{31 / 2}^{\circ} \\
a & { }^{4} \mathrm{~F}_{31 / 2}-14_{21 / 2}^{\circ} \\
a & { }^{2} \mathrm{~F}_{31 / 2}-25_{31 / 2}^{\circ} \\
a & { }^{4} \mathrm{~F}_{41 / 2}-z^{4} \mathrm{G}_{31 / 2}^{\circ}{ }^{\circ} \\
a & { }^{4} \mathrm{P}_{11 / 2}-31_{21 / 2}^{\circ}\end{array}$ & $\begin{array}{l}1560.550 \\
1562.328 \\
1562.429 \\
1563.826\end{array}$ & $\begin{array}{l}30 \\
80 \\
80 \\
20\end{array}$ & $\begin{array}{l}64080.0 \\
64007.0 \\
64002.9 \\
63945.7\end{array}$ & $\begin{array}{l}a \\
{ }^{2} \mathrm{P}_{01 / 2}-30_{01 / 2}^{\circ} \\
a^{2} \mathrm{G}_{41 / 2}-28_{31 / 2}^{\circ} \\
b^{2} \mathrm{D}_{21 / 2}-38_{11 / 2}^{\circ} \\
a \\
{ }^{4} \mathrm{~F}_{11 / 2}-9_{01 / 2}^{\circ} \\
a^{2} \mathrm{G}_{31 / 2}-31_{21 / 2}^{\circ}\end{array}$ \\
\hline $\begin{array}{l}\text { 1378. } 655 \\
1379.222 \\
1379.951 \\
1380.498 \\
1381.338\end{array}$ & $\begin{array}{r}150 \\
50 \\
150 \\
125 \\
200\end{array}$ & $\begin{array}{l}72534.5 \\
72504.6 \\
72466.3 \\
72437.6 \\
72393.6\end{array}$ & $\begin{array}{ll}a & { }^{4} \mathrm{~F}_{11 / 2}-z{ }^{4} \mathrm{~F}_{21 / 2}^{\circ} \\
a & { }^{4} \mathrm{P}_{01 / 2}-29^{\circ} 11 / 2 \\
a & { }^{4} \mathrm{P}_{21 / 2}-24_{11 / 2}^{\circ} \\
a & { }^{4} \mathrm{P}_{11 / 2}-30_{01 / 2}^{\circ} \\
a^{2} & { }^{2} \mathrm{G}_{31 / 2}-40_{31 / 2}^{0}\end{array}$ & $\begin{array}{l}1567.512 \\
1571.901 \\
1574.855 \\
1579.413\end{array}$ & $\begin{array}{r}200 \\
80 \\
200 \\
200\end{array}$ & $\begin{array}{l}\text { 63795. } 4 \\
63617.2 \\
63497.9 \\
63314.7\end{array}$ & $\begin{array}{ll}a & { }^{4} \mathrm{P}_{11 / 2}-19_{01 / 2}^{\circ} \\
a & { }^{4} \mathrm{P}_{21 / 2}-14_{21 / 2}^{\circ} \\
a & { }^{2} \mathrm{P}_{01 / 2}-29_{11 / 2}^{\circ} \\
a & { }^{4} \mathrm{~F}_{21 / 2}-z^{4} \mathrm{G}_{31 / 2}^{\circ} \\
a^{2} & { }^{2} \mathrm{~F}_{21 / 2}-21_{21 / 2}^{\circ}\end{array}$ \\
\hline $\begin{array}{l}\text { 1385. } 763 \\
1389.388 \\
1391.441 \\
1395.971 \\
1402.878\end{array}$ & $\begin{array}{l}300 \\
100 \\
180 \\
180 \\
100\end{array}$ & $\begin{array}{l}72162.4 \\
71974.1 \\
71867.9 \\
71634.7 \\
71282.0\end{array}$ & $\begin{array}{ll}a & { }^{4} \mathrm{~F}_{21 / 2}-16_{31 / 2}^{\circ} \\
a & { }^{4} \mathrm{P}_{11 / 2}-29_{11 / 2}^{\circ} \\
a & { }^{4} \mathrm{P}_{21 / 2}-23_{31 / 2}^{\circ} \\
b & { }^{2} \mathrm{D}_{11 / 2}-34_{11 / 2}^{\circ} \\
a & { }^{2} \mathrm{P}_{11 / 2}-43_{21 / 2}^{\circ}\end{array}$ & $\begin{array}{l}\text { 1580. } 277 \\
1581.226 \\
1584.074 \\
1589.559 \\
1589.680\end{array}$ & $\begin{array}{r}20 \\
20 \\
150 \\
200 \\
+\quad 80\end{array}$ & $\begin{array}{l}63280.0 \\
63242.0 \\
63128.4 \\
62910.5 \\
62905.7\end{array}$ & $\begin{array}{ll}a & { }^{2} \mathrm{~F}_{21 / 2}-20_{31 / 2}^{\circ} \\
a^{2} \mathrm{G}_{31 / 2}-28_{31 / 2}^{\circ} \\
a^{4} \mathrm{P}_{21 / 2}-13_{21 / 2}^{\circ} \\
a \quad{ }^{4} \mathrm{P}_{11 / 2}-z^{4} \mathrm{~F}_{21 / 2}^{\circ} \\
a^{4} \mathrm{~F}_{21 / 2}-z^{4} \mathrm{D}_{21 / 2}^{2}\end{array}$ \\
\hline $\begin{array}{l}\text { 1406. } 079 \\
1409.472 \\
1413.779 \\
1414.247 \\
1417.111\end{array}$ & $\begin{array}{r}20 \\
225 \\
250 \\
100 \\
100\end{array}$ & $\begin{array}{l}\text { 71119. } 7 \\
70948.5 \\
70732.4 \\
70709.0 \\
70566.1\end{array}$ & $\begin{array}{ll}a & { }^{2} \mathrm{~F}_{21 / 2}-32_{11 / 2}^{\circ} \\
a & { }^{4} \mathrm{P}_{21 / 2}-21_{21 / 2}^{\circ} \\
a & { }^{4} \mathrm{~F}_{31 / 2}-z^{4} \mathrm{~F}_{31 / 2}^{1} \\
b & { }^{2} \mathrm{D}_{21 / 2}-43_{21 / 2}^{\circ} \\
a^{4} \mathrm{~F}_{21 / 2}-z^{4} \mathrm{~F}_{11 / 2}^{\circ}\end{array}$ & $\begin{array}{l}1593.394 \\
1600.496 \\
1608.348 \\
1610.390 \\
1617.137\end{array}$ & $\begin{array}{r}150 \\
200 \\
40 \\
60 \\
250\end{array}$ & $\begin{array}{l}62759.1 \\
62480.6 \\
62175.6 \\
62096.7 \\
61837.7\end{array}$ & $\begin{array}{ll}b & { }^{2} \mathrm{D}_{11 / 2}-24_{11 / 2}^{\circ} \\
a & { }^{2} \mathrm{~F}_{31 / 2}-14_{21 / 2}^{\circ} \\
a & { }^{2} \mathrm{P}_{11 / 2}-35_{01 / 2}^{\circ} \\
a & { }^{2} \mathrm{P}_{11 / 2}-34_{11 / 2}^{\circ} \\
a & { }^{4} \mathrm{P}_{21 / 2}-12_{11 / 2}^{\circ}\end{array}$ \\
\hline $\begin{array}{l}1417.368 \\
1419.023 \\
1427.393 \\
1428.907 \\
1430.037\end{array}$ & $\begin{array}{r}125 \\
60 \\
150 \\
300 \\
250\end{array}$ & $\begin{array}{l}\text { 70553. } 3 \\
70471.0 \\
70057.8 \\
69983.6 \\
69928.3\end{array}$ & $\begin{array}{ll}a & 2 \mathrm{~F}_{31 / 2}-23_{31 / 2}^{\circ} \\
a & { }^{2} \mathrm{~F}_{21 / 2}-31_{21 / 2}^{\circ} \\
a & { }^{4} \mathrm{P}_{11 / 2}-27_{21 / 2}^{\circ} \\
a & { }^{2} \mathrm{~F}_{31 / 2}-22_{41 / 2}^{\circ} \\
a^{2} & \mathrm{G}_{31 / 2}-39_{21 / 2}^{\circ}\end{array}$ & $\begin{array}{l}\text { 1617. } 761 \\
1621.913 \\
1625.384 \\
1629.116\end{array}$ & $\begin{array}{c}100 \\
500 \\
10 \\
300 d\end{array}$ & $\begin{array}{l}61813.8 \\
61655.6 \\
61523.9 \\
61383.0\end{array}$ & $\begin{array}{l}a^{2} \mathrm{~F}_{31 / 2}-13_{21 / 2}^{\circ} \\
a^{4} \mathrm{~F}_{41 / 2}-z^{4} \mathrm{G}_{41 / 2}^{\circ} \\
b^{2} \mathrm{D}_{21 / 2}-34_{11 / 2}^{\circ} \\
a^{4} \mathrm{P}_{21 / 2}-z^{4} \mathrm{~F}_{31 / 2}^{\circ} \\
a^{4} \mathrm{~F}_{11 / 2}-z^{4} \mathrm{D}_{21 / 2}^{\circ}\end{array}$ \\
\hline $\begin{array}{l}\text { 1433. } 344 \\
1435.784 \\
1436.088 \\
1436.802 \\
1439.100\end{array}$ & $\begin{array}{r}275 \\
250 \\
80 \\
30 \\
300\end{array}$ & $\begin{array}{l}69766.9 \\
69648.3 \\
69633.6 \\
69599.0 \\
69487.9\end{array}$ & 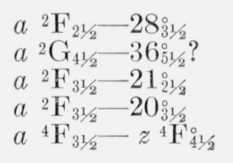 & $\begin{array}{l}1632.891 \\
1638.876 \\
1644.189 \\
1652.733 \\
1664.778\end{array}$ & $\begin{array}{r}60 \\
250 \\
100 \\
250 \\
250\end{array}$ & $\begin{array}{l}61241.1 \\
61017.6 \\
60820.3 \\
60505.8 \\
60068.1\end{array}$ & $\begin{array}{l}b^{2} \mathrm{D}_{11 / 2}-21_{21 / 2}^{\circ} \\
a^{4} \mathrm{~F}_{31 / 2}-z^{4} \mathrm{G}_{21 / 2}^{\circ}{ }^{\circ}{ }^{2}{ }^{2} \\
a^{2} \mathrm{~F}_{21 / 2}-z^{4} \mathrm{~F}_{21 / 2}^{\circ} \\
a^{2} \mathrm{G}_{41 / 2}-25_{31 / 2}^{\circ} \\
a^{2} \mathrm{~F}_{31 / 2}-z^{4} \mathrm{~F}_{31 / 2}^{\circ}\end{array}$ \\
\hline $\begin{array}{l}\text { 1441. } 173 \\
1446.334 \\
1446.701 \\
1448.393 \\
1453.173\end{array}$ & $\begin{array}{r}200 \\
150 \\
80 \\
250 \\
50\end{array}$ & $\begin{array}{l}69387.9 \\
69140.3 \\
69122.8 \\
69042.0 \\
68814.9\end{array}$ & $\begin{array}{ll}a & { }^{2} \mathrm{P}_{11 / 2}-42_{21 / 2}^{\circ} \\
a & { }^{2} \mathrm{P}_{01 / 2}-37^{\circ}{ }^{11 / 2} \\
a & { }^{4} \mathrm{P}_{01 / 2}-26_{11 / 2}^{\circ} \\
a & { }^{4} \mathrm{~F}_{11 / 2}-z^{4} \mathrm{~F}_{11 / 2}^{\circ} \\
b^{2} & { }^{2} \mathrm{D}_{21 / 2}-42_{21 / 2}^{\circ}\end{array}$ & $\begin{array}{l}\text { 1668. } 098 \\
1673.919 \\
1676.957 \\
1693.917 \\
1697.081\end{array}$ & $\begin{array}{r}100 \\
125 \\
40 \\
1000 \\
150\end{array}$ & $\begin{array}{l}59948.5 \\
59740.0 \\
59631.8 \\
59034.8 \\
58924.7\end{array}$ & $\begin{array}{l}a{ }^{4} \mathrm{P}_{01 / 2}-z^{4} \mathrm{~F}_{11 / 2}^{\circ} \\
a^{2} \mathrm{G}_{31 / 2}-25^{\circ} 1 / 2 \\
b^{2} \mathrm{D}_{11 / 2}-19_{01 / 2}^{\circ} \\
a^{4} \mathrm{~F}_{41 / 2}-z^{4} \mathrm{D}_{31 / 2}^{\circ} \\
a^{2} \mathrm{~F}_{21 / 2}-16_{31 / 2}^{\circ}\end{array}$ \\
\hline $\begin{array}{l}1454.927 \\
1462.048 \\
1464.692 \\
1471.281 \\
1473.279\end{array}$ & $\begin{array}{r}250 \\
40 \\
100 \\
150 \\
80\end{array}$ & $\begin{array}{l}\text { 68732. } 0 \\
68397.2 \\
68273.7 \\
67968.0 \\
67875.8\end{array}$ & $\begin{array}{ll}a & { }^{4} \mathrm{~F}_{21 / 2}-13_{21 / 2}^{\circ} \\
b^{2} & \mathbf{D}_{11 / 2}-31_{21 / 2}^{\circ} \\
b^{2} & \mathbf{D}_{11 / 2}-30_{01 / 2}^{\circ} \\
a^{2} & { }^{2} \mathbf{F}_{21 / 2}-27_{21 / 2}^{\circ} \\
a^{4} & { }^{4} \mathbf{F}_{11 / 2}-14_{21 / 2}^{\circ}\end{array}$ & $\begin{array}{l}1698.970 \\
1699.990 \\
1702.235 \\
1707.508 \\
1710.125\end{array}$ & $\begin{array}{l}200 \\
200 \\
200 \\
100 \\
250\end{array}$ & $\begin{array}{l}58859.2 \\
58823.9 \\
58746.3 \\
58564.9 \\
58475.2\end{array}$ & $\begin{array}{l}a{ }^{2} \mathrm{P}_{11 / 2}-31_{21 / 2}^{\circ}{ }^{\circ}{ }^{2} \mathrm{~F}_{11 / 2}-z^{4} \mathrm{~F}_{41 / 2}^{\circ} \\
b^{2} \mathrm{D}_{11 / 2}-z^{4} \mathrm{~F}_{21 / 2}^{\circ} \\
a^{2} \mathrm{P}_{01 / 2}-24_{11 / 2}^{\circ} \\
a^{2} \mathrm{G}_{41 / 2}-23_{31 / 2}^{\circ}\end{array}$ \\
\hline $\begin{array}{l}\text { 1474. } 707 \\
1481.066 \\
1482.510 \\
1482.775 \\
1487.133\end{array}$ & $\begin{array}{r}100 \\
150 \\
30 \\
30 \\
300\end{array}$ & $\begin{array}{l}67810.0 \\
67518.9 \\
67453.2 \\
67441.1 \\
67243.5\end{array}$ & $\begin{array}{ll}b^{2} \mathrm{D}_{11 / 2}-29_{11 / 2}^{\circ} \\
a & { }^{2} \mathrm{P}_{01 / 2}-35_{01 / 2}^{\circ} \\
a^{4} & \mathrm{P}_{01 / 2}-24_{11 / 2}^{\circ} \\
a^{4} \mathrm{~F}_{21 / 2}-12_{1 / 2}^{\circ} \\
a^{4} \mathrm{~F}_{31 / 2}-z^{4} \mathrm{G}_{31 / 2}^{\circ}\end{array}$ & $\begin{array}{l}1715.670 \\
1716.697 \\
1717.820 \\
1726.952 \\
1727.281\end{array}$ & $\begin{array}{r}200 \\
100 \\
300 \\
5 \\
500\end{array}$ & $\begin{array}{l}58286.3 \\
58251.4 \\
58213.3 \\
57905.5 \\
57894.5\end{array}$ & $\begin{array}{l}b{ }^{2} \mathrm{D}_{21 / 2}-31_{21 / 2}^{\circ} \\
a \\
{ }^{4} \mathrm{P}_{11 / 2}-14_{21 / 2}^{\circ} \\
a \\
{ }^{4} \mathrm{~F}_{11 / 2}-z^{4} \mathrm{D}_{01 / 2}^{\circ} \\
a^{2} \mathrm{G}_{41 / 2}-22_{41 / 2}^{\circ} \\
a^{4} \mathrm{P}_{21 / 2}^{\circ}-z^{4} \mathrm{G}_{31 / 2}^{\circ}\end{array}$ \\
\hline $\begin{array}{l}1487.906 \\
1489.446 \\
1492.829 \\
1494.266 \\
1500.334\end{array}$ & $\begin{array}{r}250 \\
200 \\
\left(\begin{array}{r} \\
20 \\
250\end{array}\right.\end{array}$ & $\begin{array}{l}67208.5 \\
67139.0 \\
66986.9 \\
66922.5 \\
66651.8\end{array}$ & $\begin{array}{ll}a^{4} \mathrm{~F}_{11 / 2}-13_{21 / 2}^{\circ} \\
a & { }^{2} \mathrm{~F}_{31 / 2}-z^{4} \mathrm{~F}_{21 / 2}^{\circ} \\
a^{4} \mathrm{~F}_{21 / 2}-z^{4} \mathrm{~F}_{31 / 2}^{\circ} \\
a^{4} \mathrm{P}_{11 / 2}-24_{11 / 2}^{\circ} \\
a^{4} \mathrm{~F}_{31 / 2}-z^{4} \mathrm{D}_{21 / 2}^{\circ}\end{array}$ & $\begin{array}{l}\text { 1733. } 140 \\
1736.590 \\
1738.484 \\
1744.346 \\
1745.098\end{array}$ & $\begin{array}{r}100 d \\
60 \\
300 \\
150 \\
40\end{array}$ & $\begin{array}{l}57698.9 \\
57584.1 \\
57521.4 \\
57328.1 \\
57303.4\end{array}$ & $\begin{array}{l}b^{2} \mathrm{D}_{21 / 2}-29_{11 / 2}^{\circ} \\
a^{4} \mathrm{P}_{11 / 2}-13_{21 / 2}^{\circ} \\
a^{2} \mathrm{G}_{11 / 2}-20_{31 / 2}^{\circ} \\
a^{2}{ }^{2} \mathrm{~F}_{21 / 2}-z^{4} \mathrm{~F}_{11 / 2}^{\circ} \\
a^{4} \mathrm{P}_{21 / 2}-z^{4} \mathrm{D}_{21 / 2}^{\circ}\end{array}$ \\
\hline
\end{tabular}


Table 1. Identified lines of Au III-Continued

\begin{tabular}{|c|c|c|c|c|c|c|c|}
\hline$\lambda$ (vac.) & $\begin{array}{l}\text { Intensity } \\
\text { and } \\
\text { character }\end{array}$ & Wave No. & Combination & $\lambda$ (vac.) & $\begin{array}{l}\text { Intensity } \\
\text { and } \\
\text { character }\end{array}$ & Wave No. & Combination \\
\hline $\begin{array}{c}A \\
1746.037 \\
1750.095 \\
1756.917 \\
1759.800 \\
1760.881\end{array}$ & $\begin{array}{r}500 \\
30 \\
500 \\
20 \\
60\end{array}$ & $\begin{array}{l}\mathrm{cm}^{-1} \\
57272.5 \\
57139.9 \\
56917.9 \\
56824.6 \\
56789.7\end{array}$ & $\begin{array}{l}a{ }^{4} \mathrm{~F}_{21 / 2}-z^{4} \mathrm{G}_{21 / 2}^{\circ} \\
a^{2} \mathrm{G}_{31 / 2}-22_{41 / 2}^{\circ} \\
a^{4} \mathrm{~F}_{21 / 2}^{\circ}-z^{4} \mathrm{D}_{11 / 2}^{\circ} \\
a^{4} \mathrm{P}_{01 / 2}^{\circ}-12_{11 / 2}^{\circ} \\
a^{2} \mathrm{G}_{31 / 2}^{\circ}-21_{21 / 2}^{\circ}\end{array}$ & $\begin{array}{c}A \\
\text { 1989. } 631 \\
\text { 1996. } 853 \\
\text { 2041. } 435 \\
\text { 2055. } 459 \\
\text { 2083. } 092\end{array}$ & $\begin{array}{r}400 \\
150 \\
60 \\
60 \\
300\end{array}$ & $\begin{array}{l}c m^{-1} \\
50260.6 \\
50078.8 \\
48969.4 \\
48635.4 \\
47990.3\end{array}$ & $\begin{array}{l}a{ }^{2} \mathrm{~F}_{21 / 2}-z^{4} \mathrm{G}_{31 / 2}^{\circ} \\
a^{2} \mathrm{P}_{11 / 2}-18_{01 / 2}^{\circ} \\
a^{2} \mathrm{G}_{31 / 2}-13_{21 / 2}^{\circ} \\
b^{2} \mathrm{D}_{21 / 2}-z^{4} \mathrm{~F}_{21 / 2}^{\circ} \\
a^{2} \mathrm{G}_{41 / 2}-z^{4} \mathrm{~F}_{31 / 2}^{\circ}\end{array}$ \\
\hline $\begin{array}{l}1761.947 \\
1767.415 \\
1774.419 \\
1775.166 \\
1776.396\end{array}$ & $\begin{array}{l}500 \\
300 \\
100 \\
800 \\
200\end{array}$ & $\begin{array}{l}56755.4 \\
56579.8 \\
56356.5 \\
56332.8 \\
56293.8\end{array}$ & $\begin{array}{l}a^{2} \mathrm{G}_{31 / 2}-20_{31 / 2}^{\circ} \\
a a^{2} \mathrm{~F}_{31 / 2}-z^{4} \mathrm{G}_{31 / 2}^{\circ} \\
a^{2} \mathrm{P}_{11 / 2}-27_{21 / 2}^{\circ} \\
a^{4} \mathrm{~F}_{31 / 2}-z^{4} \mathrm{G}_{41 / 2}^{\circ} \\
a^{4} \mathrm{P}_{11 / 2}-12_{11 / 2}^{\circ}\end{array}$ & $\begin{array}{l}2085.452 \\
2100.392 \\
2116.879 \\
2159.085 \\
2167.332\end{array}$ & $\begin{array}{r}80 \\
20 \\
2 \\
100 \\
80\end{array}$ & $\begin{array}{l}\text { 47936. } 0 \\
47595.0 \\
47224.4 \\
46301.2 \\
46125.2\end{array}$ & $\begin{array}{ll}a & { }^{2} \mathrm{P}_{01 / 2}-12_{11 / 2}^{\circ} \\
b & { }^{2} \mathrm{D}_{11 / 2}-z^{4} \mathrm{D}_{21 / 2}^{\circ} \\
a^{2} \mathrm{G}_{31 / 2}-z^{4} \mathrm{~F}_{31 / 2}^{\circ} & \circ{ }^{2} \\
a & { }^{4} \mathrm{P}_{01 / 2}-z^{4} \mathrm{D}_{11 / 2}^{\circ} \\
a^{4}{ }^{4} \mathrm{P}_{11 / 2}-z^{4} \mathrm{G}_{21 / 2}^{\circ}\end{array}$ \\
\hline $\begin{array}{l}1780.571 \\
1786.106 \\
1792.653 \\
1793.762 \\
1801.982\end{array}$ & $\begin{array}{l}100 \\
300 \\
150 \\
500 \\
200\end{array}$ & $\begin{array}{l}56161.8 \\
55987.7 \\
55783.2 \\
55748.8 \\
55494.4\end{array}$ & $\begin{array}{l}a{ }^{2} \mathrm{~F}_{21 / 2}-14_{21 / 2}^{\circ} \\
a^{2} \mathrm{~F}_{31 / 2}^{\circ}-z^{4} \mathrm{D}_{21 / 2}^{\circ} \\
b^{2} \mathrm{D}_{21 / 2}-27_{21 / 2}^{\circ} \\
a^{4} \mathrm{~F}_{11 / 2}^{\circ}-z^{4} \mathrm{G}_{21 / 2}^{\circ} \\
a^{2}{ }^{2} \mathrm{~F}_{21 / 2}-13_{21 / 2}^{\circ}\end{array}$ & $\begin{array}{l}2172.200 \\
2184.108 \\
2186.673 \\
2188.966 \\
2253.448\end{array}$ & $\begin{array}{r}200 \\
100 \\
50 \\
500 \\
40\end{array}$ & $\begin{array}{l}46021.8 \\
45770.9 \\
45717.3 \\
45669.4 \\
44362.7\end{array}$ & $\begin{array}{ll}a & { }^{2} \mathrm{P}_{01 / 2}-9_{01 / 2}^{\circ} \\
a & { }^{4} \mathrm{P}_{11 / 2}-z^{4} \mathrm{D}_{11 / 2}^{\circ}{ }^{11 / 2} \\
a & { }^{2} \mathrm{P}_{11 / 2}-z^{4} \mathrm{~F}_{11 / 2}^{\circ} \\
a^{2} & { }^{2} \mathrm{~F}_{31 / 2}-z^{4} \mathrm{G}_{41 / 2}^{\circ} \\
a^{4}{ }^{4} \mathrm{P}_{21 / 2}-z^{4} \mathrm{D}_{31 / 2}^{\circ}\end{array}$ \\
\hline $\begin{array}{l}1805.235 \\
1809.811 \\
1821.169 \\
1821.801 \\
1841.019\end{array}$ & $\begin{array}{r}400 \\
100 \\
400 \\
20 \\
20\end{array}$ & $\begin{array}{l}55394.4 \\
55254.4 \\
54909.8 \\
54890.7 \\
54317.7\end{array}$ & $\begin{array}{l}a{ }^{4} \mathrm{~F}_{11 / 2}-z^{4} \mathrm{D}_{11 / 2}^{\circ} \\
b^{2} \mathrm{D}_{11 / 2}-z^{4} \mathrm{~F}_{11 / 2}^{11 / 2} \\
a^{4} \mathrm{P}_{01 / 2}-9_{01 / 2}^{\circ} \\
a^{2} \mathrm{P}_{11 / 2}-26_{11 / 2}^{\circ} \\
b^{2} \mathrm{D}_{21 / 2}-26_{11 / 2}^{\circ}\end{array}$ & $\begin{array}{l}2270.217 \\
2278.045 \\
2288.626 \\
2308.200 \\
2322.267\end{array}$ & $\begin{array}{r}10 \\
5 \\
40 \\
10 \\
300\end{array}$ & $\begin{array}{l}\text { 44035. } 2 \\
43883.7 \\
43680.8 \\
43310.5 \\
43048.1\end{array}$ & $\begin{array}{l}a^{2} \mathrm{~F}_{21 / 2}-z^{4} \mathrm{G}_{21 / 2}^{\circ} \\
a^{2} \mathrm{P}_{11 / 2}-13_{21 / 2}^{\circ} \\
a^{2} \mathrm{~F}_{21 / 2}-z^{4} \mathrm{D}_{11 / 2}^{\circ} \\
b^{2} \mathrm{D}_{21 / 2}-13_{21 / 2}^{\circ} \\
a^{2} \mathrm{~F}_{31 / 2}^{\circ}-z^{4} \mathrm{D}_{31 / 2}^{\circ}\end{array}$ \\
\hline $\begin{array}{l}\text { 1844. } 889 \\
1848.833 \\
1849.088 \\
1860.484 \\
1861.799\end{array}$ & $\begin{array}{r}400 \\
150 \\
50 \\
40 \\
500\end{array}$ & $\begin{array}{l}54203.8 \\
54088.2 \\
54080.7 \\
53749.5 \\
53711.5\end{array}$ & $\begin{array}{l}a{ }^{2} \mathrm{~F}_{21 / 2}-12_{11 / 2}^{\circ} \\
b^{2} \mathrm{D}_{11 / 2}^{\circ}-14_{21 / 2}^{\circ} \\
b^{2} \mathrm{D}_{21 / 2}-25_{31 / 2}^{\circ} \\
a^{2} \mathrm{~F}_{21 / 2}-z^{4} \mathrm{~F}_{31 / 2}^{\circ} \\
a^{4} \mathrm{~F}_{31 / 2}-z^{4} \mathrm{D}_{31 / 2}^{\circ}\end{array}$ & $\begin{array}{l}2347.105 \\
2379.106 \\
2382.403 \\
2402.706 \\
2405.118\end{array}$ & $\begin{array}{r}20 \\
10 \\
100 \\
150 \\
150\end{array}$ & $\begin{array}{l}42592.6 \\
42019.8 \\
41961.6 \\
41607.1 \\
41565.3\end{array}$ & $\begin{array}{l}a{ }^{2} \mathrm{P}_{11 / 2}-12_{11 / 2}^{\circ} \\
b^{2} \mathrm{D}_{21 / 2}-12_{11 / 2}^{\circ} \\
b^{2} \mathrm{D}_{11 / 2}-z^{4} \mathrm{G}_{21 / 2}^{\circ}{ }^{21 / 2} \\
b^{2} \mathrm{D}_{11 / 2}-z^{4} \mathrm{D}_{11 / 2}^{\circ}{ }^{2}{ }^{2}{ }^{2} \mathrm{D}_{21 / 2}-z^{4} \mathrm{~F}_{31 / 2}^{\circ}\end{array}$ \\
\hline $\begin{array}{l}\text { 1871. } 922 \\
1880.911 \\
\text { 1899. } 405 \\
\text { 1918. } 278 \\
\text { 1932. } 038\end{array}$ & $\begin{array}{r}150 \\
30 \\
60 \\
150 \\
100\end{array}$ & $\begin{array}{l}53421.0 \\
53165.7 \\
52648.1 \\
52130.1 \\
51758.8\end{array}$ & $\begin{array}{l}b^{2} \mathrm{D}_{11 / 2}-13_{21 / 2}^{\circ} \\
a^{2} \mathrm{G}_{41 / 2}-16_{31 / 2}^{\circ} \\
b^{2} \mathrm{D}_{21 / 2}-24_{11 / 2}^{\circ} \\
b^{2} \mathrm{D}_{111 / 2}-12_{11 / 2}^{\circ} \\
a^{4} \mathrm{P}_{11 / 2}^{\circ}-z^{4} \mathrm{D}_{21 / 2}^{\circ}\end{array}$ & $\begin{array}{l}2625.522 \\
2665.159 \\
2666.994 \\
2721.835 \\
3117.339\end{array}$ & $\begin{array}{l}10 d \\
10 \\
10 \\
50 \\
10\end{array}$ & $\begin{array}{l}38076.3 \\
37510.1 \\
37484.3 \\
36729.0 \\
32069.3\end{array}$ & $\begin{array}{l}b^{2} \mathrm{D}_{21 / 2}-z^{4} \mathrm{G}_{31 / 2}^{\circ} \\
a^{2} \mathrm{G}_{31 / 2}-z^{4} \mathrm{G}_{21 / 2}^{0} \\
b^{2} \mathrm{D}_{21 / 2}-z^{4} \mathrm{D}_{21 / 2}^{\circ} \\
a^{2} \mathrm{~F}_{21 / 2}-z^{4} \mathrm{D}_{31 / 2}^{2}{ }^{2}{ }^{2}{ }^{2} \mathrm{P}_{11 / 2}-z^{4} \mathrm{D}_{11 / 2}^{\circ}\end{array}$ \\
\hline $\begin{array}{l}\text { 1934. } 114 \\
\text { 1935. } 416 \\
\text { 1948. } 792 \\
1958.472 \\
1985.951\end{array}$ & $\begin{array}{c}60 \\
100 \\
200 \\
100 \\
20 d\end{array}$ & $\begin{array}{l}51703.3 \\
51668.2 \\
51313.8 \\
51060.2 \\
50353.7\end{array}$ & $\begin{array}{ll}a & { }^{2} \mathrm{P}_{11 / 2}-21_{21 / 2}^{\circ} \\
a & { }^{4} \mathrm{P}_{21 / 2}-z^{4} \mathrm{G}_{21 / 2} \\
a & { }^{4} \mathrm{P}_{21 / 2}-z^{4} \mathrm{D}_{11 / 2}^{\circ} \\
a^{2} \mathrm{P}_{01 / 2}-z^{4} \mathrm{~F}_{11 / 2}^{\circ} \\
a^{2} \mathrm{~F}_{31 / 2}-z^{4} \mathrm{G}_{21 / 2}^{\circ}\end{array}$ & $\begin{array}{l}\text { 3138. } 730 \\
3174.057 \\
3227.991 \\
3309.856\end{array}$ & $\begin{array}{r}10 \\
20 \\
100 \\
100\end{array}$ & $\begin{array}{l}31850.8 \\
31496.3 \\
30970.0 \\
30204.1\end{array}$ & $\begin{array}{l}b^{2} \mathrm{D}_{21 / 2}-z^{4} \mathrm{G}_{21 / 2}^{0} \\
b^{2} \mathrm{D}_{21 / 2}-z^{4} \mathrm{D}_{11 / 2}^{\circ} \\
a^{2} \mathrm{G}_{41 / 2}-z^{4} \mathrm{D}_{31 / 2}^{\circ} \\
a^{2} \mathrm{G}_{31 / 2}-z^{4} \mathrm{D}_{31 / 2}^{\circ}\end{array}$ \\
\hline
\end{tabular}

NOTE. $-h=$ hazy; $w=$ wide; $(\quad)=$ masked $b_{y}$ another line; $d=$ double.

TABLE 2. Fven levele of $\mathrm{Au}$ III

\begin{tabular}{|c|c|c|c|c|c|c|c|c|c|}
\hline Electron structure & $\begin{array}{c}\text { Possible } \\
\text { desig- } \\
\text { nation }\end{array}$ & $J$ & Level & Interval & Electron structure & $\begin{array}{l}\text { Possible } \\
\text { desig- } \\
\text { nation }\end{array}$ & $J$ & Level & Interval \\
\hline $\begin{array}{l}5 d^{9} \\
5 d^{9} \\
5 d^{8}\left({ }^{3} \mathrm{~F}_{4}\right) 6 s \\
5 d^{8}\left({ }^{3} \mathrm{~F}_{4}\right) 6 s \\
5 d^{8}\left({ }^{3} \mathrm{~F}_{3}\right) 6 s \\
5 d^{8}\left({ }^{3} \mathrm{~F}_{2}\right) 6 s \\
5 d^{8}\left({ }^{3} \mathrm{P}_{2}\right) 6 s \\
5 d^{8}\left({ }^{3} \mathrm{~F}_{3}\right) 6 s\end{array}$ & $\begin{array}{l}a^{2} \mathrm{D} \\
a^{2} \mathrm{D} \\
a^{4} \mathrm{~F} \\
a^{4} \mathrm{~F} \\
a^{4} \mathrm{~F} \\
a^{4} \mathrm{~F}\end{array}$ & $\begin{array}{l}21 / 2 \\
11 / 2 \\
41 / 2 \\
31 / 2 \\
21 / 2 \\
11 / 2 \\
21 / 2 \\
31 / 2\end{array}$ & $\begin{array}{r}0.0 \\
12694.0 \\
\\
29753.6 \\
35076.7 \\
38822.2 \\
40345.6 \\
44425.9 \\
45740.5\end{array}$ & $\begin{array}{l}-12694.0 \\
-5323.1 \\
-3745.5 \\
-1523.4\end{array}$ & $\begin{array}{l}5 d^{8}\left({ }^{3} \mathrm{P}_{1}\right) 6 s \\
5 d^{8}\left({ }^{3} \mathrm{P}_{2}\right) 6 s \\
5 d^{8}\left({ }^{3} \mathrm{~F}_{2}\right) 6 s \\
5 d^{8}\left({ }^{1} \mathrm{D}_{2}\right) 6 s \\
5 d^{8}\left({ }^{1} \mathrm{G}_{4}\right) 6 s \\
5 d^{8}\left({ }^{3} \mathrm{P}_{0}\right) 6 s \\
5 d^{8}\left({ }^{1} \mathrm{G}_{4}\right) 6 s \\
5 d^{8}\left({ }^{3} \mathrm{P}_{1}\right) 6 s \\
5 d^{8}\left({ }^{1} \mathrm{D}_{2}\right) 6 s\end{array}$ & $\begin{array}{l}a^{4} \mathrm{P} \\
a^{4} \mathrm{P} \\
a^{2} \mathrm{~F} \\
b^{2} \mathrm{D} \\
a^{2} \mathrm{G} \\
a^{2} \mathrm{P} \\
a^{2} \mathrm{G} \\
a^{2} \mathrm{P} \\
b^{2} \mathrm{D}\end{array}$ & $\begin{array}{l}01 / 2 \\
11 / 2 \\
21 / 2 \\
11 / 2 \\
41 / 2 \\
01 / 2 \\
31 / 2 \\
11 / 2 \\
21 / 2\end{array}$ & $\begin{array}{l}49438.9 \\
49969.4 \\
52059.6 \\
54133.2 \\
57818.6 \\
58327.1 \\
58584.6 \\
63670.9 \\
64244.0\end{array}$ & \\
\hline
\end{tabular}


TABLE 3. Odd levels of Au III

\begin{tabular}{|c|c|c|c|c|c|c|c|c|c|}
\hline Electron structure & $\begin{array}{c}\text { Possible } \\
\text { desig- } \\
\text { nation }\end{array}$ & $\begin{array}{c}\text { Num- } \\
\text { ber }\end{array}$ & $J$ & Level & Electron structure & $\begin{array}{c}\text { Possible } \\
\text { desig- } \\
\text { nation }\end{array}$ & $\begin{array}{c}\text { Num- } \\
\text { ber }\end{array}$ & $J$ & Level \\
\hline $\begin{array}{l}5 d^{8}\left({ }^{3} \mathrm{~F}\right) 6 p \\
5 d^{8}\left({ }^{3} \mathrm{~F}\right) 6 p \\
5 d^{8}\left({ }^{3} \mathrm{~F}\right) 6 p \\
5 d^{8}\left({ }^{3} \mathrm{~F}\right) 6 p \\
5 d^{8}\left({ }^{3} \mathrm{~F}\right) 6 p \\
5 d^{8}\left({ }^{3} \mathrm{~F}\right) 6 p \\
5 d^{8}\left({ }^{3} \mathrm{~F}\right) 6 p \\
\left.5 d^{8}{ }^{3} \mathrm{~F}\right) 6 p \\
5 d^{8}\left({ }^{3} \mathrm{P}\right) 6 p \\
\left.5 d^{8}{ }^{3} \mathrm{~F}\right) 6 p \\
5 d^{8}\left({ }^{3} \mathrm{~F}\right) 6 p \\
\left.5 d^{8}{ }^{3} \mathrm{P}\right) 6 p \\
\left.5 d^{8}{ }^{3} \mathrm{P}\right) 6 p \\
5 d^{8}\left({ }^{3} \mathrm{~F}\right) 6 p \\
5 d^{8}\left({ }^{3} \mathrm{~F}\right) 6 p \\
5 d^{8}\left({ }^{3} \mathrm{P}\right) 6 p \\
\left.3 d^{8}{ }^{3} \mathrm{~F}\right) 6 p \\
\left.5 d^{8}{ }^{3} \mathrm{P}\right) 6 p \\
5 d^{8}\left({ }^{1} \mathrm{D}\right) 6 p \\
5 d^{8}\left({ }^{1} \mathrm{G}\right) 6 p \\
5 d^{8}\left({ }^{3} \mathrm{~F}\right) 6 p \\
5 d^{8}\left({ }^{3} \mathrm{~F}\right) 6 p \\
5 d^{8}\left({ }^{3} \mathrm{~F}\right) 6 p\end{array}$ & $\begin{array}{l}z^{4} \mathrm{D}^{\circ} \\
z^{4} \mathrm{G}^{\circ} \\
z^{4} \mathrm{D}^{\circ} \\
z^{4} \mathrm{G}^{\circ} \\
z^{4} \mathrm{D}^{\circ} \\
z^{4} \mathrm{D}^{\circ} \\
z^{4} \mathrm{G}^{\circ} \\
z^{4} \mathrm{G}^{\circ} \\
{ }^{4} \mathrm{P}^{\circ} \\
z^{4} \mathrm{~F}^{\circ} \\
z^{4} \mathrm{~F}^{\circ} \\
{ }^{4} \mathrm{P}^{\circ} \\
{ }^{4} \mathrm{P}^{\circ} \\
{ }^{2} \mathrm{D}^{\circ} \\
z^{4} \mathrm{~F}^{\circ} \\
{ }^{4} \mathrm{D}^{\circ} \\
z^{4} \mathrm{~F}^{\circ} \\
{ }^{2} \mathrm{~S}^{\circ} \\
{ }^{2} \mathrm{P}^{\circ} \\
{ }^{2} \mathrm{~F}^{\circ} \\
{ }^{2} \mathrm{~F}^{\circ} \\
{ }^{2} \mathrm{G}^{\circ} \\
{ }^{2} \mathrm{~F}^{\circ}\end{array}$ & $\begin{array}{l}1^{\circ} \\
2^{\circ} \\
3^{\circ} \\
4^{\circ} \\
5^{\circ} \\
6^{\circ} \\
7^{\circ} \\
8^{\circ} \\
9^{\circ} \\
10^{\circ} \\
11^{\circ} \\
12^{\circ} \\
13^{\circ} \\
14^{\circ} \\
15^{\circ} \\
16^{\circ} \\
17^{\circ} \\
18^{\circ} \\
19^{\circ} \\
20^{\circ} \\
21^{\circ} \\
22^{\circ} \\
23^{\circ}\end{array}$ & $\begin{array}{l}31 / 2 \\
41 / 2 \\
11 / 2 \\
21 / 2 \\
01 / 2 \\
21 / 2 \\
31 / 2 \\
51 / 2 \\
01 / 2 \\
41 / 2 \\
31 / 2 \\
11 / 2 \\
21 / 2 \\
21 / 2 \\
11 / 2 \\
31 / 2 \\
21 / 2 \\
01 / 2 \\
01 / 2 \\
31 / 2 \\
21 / 2 \\
41 / 2 \\
31 / 2\end{array}$ & $\begin{array}{r}88788.5 \\
91409.4 \\
95740.0 \\
96094.5 \\
98559.1 \\
101728.2 \\
102320.2 \\
102993.7 \\
104348.3 \\
104564.6 \\
105809.1 \\
106263.1 \\
107554.2 \\
108221.2 \\
109387.6 \\
110984.1 \\
112879.6 \\
113749.9 \\
113764.9 \\
115339.9 \\
115374.2 \\
115724.2 \\
116293.8\end{array}$ & $\begin{array}{l}5 d^{8}\left({ }^{3} \mathrm{P}\right) 6 p \\
5 d^{8}\left({ }^{1} \mathrm{D}\right) 6 p \\
5 d^{8}\left({ }^{3} \mathrm{~F}\right) 6 p \\
5 d^{8}\left({ }^{3} \mathrm{P}\right) 6 p \\
5 d^{8}\left({ }^{3} \mathrm{~F}\right) 6 p \\
5 d^{8}\left({ }^{1} \mathrm{D}\right) 6 p \\
5 d^{8}\left({ }^{3} \mathrm{P}\right) 6 p \\
5 d^{8}\left({ }^{1} \mathrm{D}\right) 6 p \\
\left.5 d^{8}{ }^{3} \mathrm{P}\right) 6 p \\
5 d^{8}(1 \mathrm{G}) 6 p \\
5 d^{8}\left({ }^{1} \mathrm{D}\right) 6 p \\
5 d^{8}\left({ }^{3} \mathrm{P}\right) 6 p \\
5 d^{8}\left({ }^{1} \mathrm{G}\right) 6 p \\
5 d^{8}\left({ }^{3} \mathrm{P}\right) 6 p \\
5 d^{8}\left({ }^{3} \mathrm{P}\right) 6 p \\
5 d^{8}\left({ }^{1} \mathrm{G}\right) 6 p \\
5 d^{8}(1 \mathrm{G}) 6 p \\
5 d^{8}\left({ }^{1} \mathrm{G}\right) 6 p \\
5 d^{8}\left({ }^{3} \mathrm{P}\right) 6 p \\
5 d^{8}\left({ }^{1} \mathrm{D}\right) 6 p \\
5 d^{7} 6 s 6 p \\
5 d^{7} 6 s 6 p\end{array}$ & $\begin{array}{l}{ }^{4} \mathrm{~S}^{\circ} \\
{ }^{2} \mathrm{~F}^{\circ} \\
{ }^{2} \mathrm{D}^{\circ} \\
{ }^{4} \mathrm{D}^{\circ} \\
{ }^{2} \mathrm{G}^{\circ} \\
{ }^{2} \mathrm{P}^{\circ} \\
{ }^{4} \mathrm{D}^{\circ} \\
{ }^{2} \mathrm{~F}^{\circ} \\
{ }^{4} \mathrm{D}^{\circ} \\
{ }^{2} \mathrm{H}^{\circ} \\
{ }^{2} \mathrm{D}^{\circ} \\
{ }^{2} \mathrm{P}^{\circ} \\
{ }^{2} \mathrm{H}^{\circ} \\
{ }^{2} \mathrm{D}^{\circ} \\
{ }^{2} \mathrm{P}^{\circ} \\
{ }^{2} \mathrm{~F}^{\circ} \\
{ }^{2} \mathrm{G}^{\circ} \\
{ }^{2} \mathrm{G}^{\circ} \\
{ }^{2} \mathrm{D}^{\circ} \\
{ }^{2} \mathrm{D}^{\circ}\end{array}$ & $\begin{array}{l}24^{\circ} \\
25^{\circ} \\
26^{\circ} \\
27^{\circ} \\
28^{\circ} \\
29^{\circ} \\
30^{\circ} \\
31^{\circ} \\
32^{\circ} \\
33^{\circ} \\
34^{\circ} \\
35^{\circ} \\
36^{\circ} \\
37^{\circ} \\
38^{\circ} \\
39^{\circ} \\
40^{\circ} \\
41^{\circ} \\
42^{\circ} \\
43^{\circ} \\
44^{\circ} \\
45^{\circ}\end{array}$ & $\begin{array}{l}11 / 2 \\
31 / 2 \\
11 / 2 \\
21 / 2 \\
31 / 2 \\
11 / 2 \\
01 / 2 \\
21 / 2 \\
11 / 2 \\
41 / 2 \\
11 / 2 \\
01 / 2 \\
51 / 2 \\
11 / 2 \\
11 / 2 \\
21 / 2 \\
31 / 2 \\
41 / 2 \\
21 / 2 \\
21 / 2 \\
31 / 2 \\
21 / 2 \\
2\end{array}$ & $\begin{array}{l}116892.1 \\
118324.6 \\
118561 . \% \\
120027.3 \\
121826.4 \\
121943.5 \\
12240 \% .0 \\
122530.3 \\
123179.0 \\
123508.8 ? \\
125767.9 \\
125846.2 \\
127467.0 ? \\
12746 \% .6 \\
128250.9 \\
128512.7 \\
130978.2 \\
132353.0 \\
133058.9 \\
134953.0 \\
156532.0 \\
158323.1\end{array}$ \\
\hline
\end{tabular}

(Paper 64A6-69) 